\title{
CAPACIDADE ANTIOXIDANTE DE INFUSÕES DE CAFÉS DESCAFEINADOS E EPILEPSIA
}

\author{
Autores: Leandro Galhardi Paez ${ }^{1}$, Daniel Rettori ${ }^{3}$, Ivair Donizeti Gonçalves ${ }^{1}$, Susana \\ Nogueria Diniz ${ }^{1,2}$, Regina Mara Silva Pereira ${ }^{1,2^{*}}$ \\ ${ }^{1}$ Programa de Mestrado Profissional em Farmácia - Universidade Anhanguera de \\ São Paulo (UNIAN-SP), São Paulo, Brasil \\ ${ }^{2}$ Programa de Pós-graduação em Biotecnologia e Inovação em Saúde -- \\ Universidade Anhanguera de São Paulo (UNIAN-SP), São Paulo, SP, Brasil \\ ${ }^{3}$ Universidade Federal de São Paulo (UNIFESP), São Paulo, SP, Brasil \\ *Endereço de correspondência: rpereira02@hotmail.com. Av. Raimundo Pereira de \\ Magalhães, 3305, Pirituba, São Paulo, SP, Brasil. CEP 05145-200, TEl: \\ 55(11)35128415
}

Recebido em: 22/09/2018 - Aprovado em: 23/11/2018 - Publicado em: 03/12/2018 DOI: 10.18677/EnciBio_2018B118

\begin{abstract}
RESUMO
Epilepsia é uma doença crônica neurodegenerativa, que se caracteriza por convulsões recorrentes, levando à produção excessiva de radicais livres, e sua progressão está associada ao estresse oxidativo. $O$ estresse oxidativo consiste em um desequilíbrio persistente entre a produção de espécies oxidantes reativas (EOR) e as defesas antioxidantes, resultando muitas vezes em danos celulares irreversíveis. O café é uma bebida rica em antioxidantes naturais podendo apresentar propriedades antioxidantes relevantes. Entretanto, a cafeina também presente no café tem um efeito danoso aos portadores de eplepsia, pois a sua sobredosagem pode aumentar as crises epiléticas. Neste trabalho avaliou-se 13 amostras de cafés (sete de cáfes de cultivo convensional, três descafeinados e três de cultivo orgânicos) para avaliar a capacidade antioxidante (CA) e investigar se a ausência de cafeina nas amostras afetaria a CA dos cafés. Foi utilizado o método de supressão de radicais ABTS.- para determinar a capacidade de remover $50 \%$ dos radicais de ABTS $^{\circ}\left(\mathrm{CA}_{50}\right)$. Para a análise estatística foi utilizado o teste ANOVA. Os cafés descafeinados apresentaram CA similar aos dos cafés que contem cafeina, tanto de cultivo tradicional quanto orgânico, demonstrando que a cafeina não afeta diretamente a CA da bebida, o que permite que portadores de epilepsia usufruam de seu consumo e benefícios terapeuticos.
\end{abstract}

PALAVRAS-CHAVE: antioxidante, café, epilepsia.

\section{ANTIOXIDANT CAPACITY OF COFFEE INFUSIONS AND EPILEPSY}

\begin{abstract}
Epilepsy is a chronic neurodegenerative disease, characterized by recurrent seizures, leading to excessive free radical production, and its progression is associated with oxidative stress. Oxidative stress consists of a persistent imbalance between the production of reactive oxidant species (EOR) and antioxidant defenses, often resulting in irreversible cellular damage. Coffee is a beverage rich in natural antioxidants and may have relevant antioxidant properties. However, caffeine also ENCICLOPÉDIA BIOSFERA, Centro Científico Conhecer - Goiânia, v.15 n.28; p.1202 2018
\end{abstract}


present in coffee has a damaging effect on epilepsy patients, and their overdose may increase epileptic seizures. In this work 13 coffee samples (7 of conventional, 3 decaffeinated and 3 organic culture heads) were analyzed to evaluate the antioxidant capacity (AC) and to investigate if the absence of caffeine in the samples decaffeinated would affect the AC. The ABTS radical suppression method was used to determine the ability to remove $50 \%$ ABTS $^{\circ}\left(\mathrm{CA}_{50}\right)$ radicals. To determinate statistical differences was used ANOVA. Decaffeinated coffee presented AC like coffees containing caffeine, both traditional and organic, demonstrating that caffeine does not directly affect the AC of the beverage, which allows epilepsy patients to enjoy its consumption and therapeutic benefits.

KEYWORDS: antioxidant, coffee, epilepsies.

\section{INTRODUÇÃO}

Epilepsia é uma doença crônica, que se caracteriza por convulsões recorrentes e demais complicações, levando ao desequilíbrio entre a concentração de radicais livres e agentes redutores no cérebro gerando estresse oxidativo (PUTTACHARY et al., 2015). O estresse oxidativo é definido como um desequilíbrio persistente entre a produção de espécies oxidantes reativas (EOR) e as defesas antioxidantes do organismo humano (PUTTACHARY et al., 2015), resultando muitas vezes em danos celulares irreversíveis. Na epilepsia esse desequilíbrio é constatado pelo aumento de marcadores de estresse oxidativo, como o óxido nítrico (NO) e malondialdeído (MDA) (CHUNG et al., 2011). O estresse oxidativo resultante da geração excessiva dessas espécies tem sido implicado na iniciação, bem como a progressão da epilepsia e de outras doenças neurodegenerativas, como Alzheimer e Parkinson (SHICHIN et al., 2014; PRASAD et al, 2017). Os efeitos pró oxidativos proveniente do uso crônico de medicamentos antiepiléticos (AEDs) podem levar ao estresse oxidativo adicional, aumento de convulsões, resultando na perda de eficiência de algumas drogas como ácido valproico, fenitoína e carbamazepina (SHICHIN et al., 2014). O uso concomitante de antioxidantes e AEDs convencionais poderá ter um efeito benéfico no tratamento da epilepsia.

O café por ser rico em polifenóis é uma importante fonte de compostos antioxidantes naturais na dieta dos seres humanos (YESIL e YILMAZ, 2013; NIEMAN et al., 2018;). Esses polifenóis inibem a nicotinamida adenina dinucleótido fosfato oxidase (NADPH) na mitocôndria e diminui a produção de espécies reativas de oxigênio (ROS) (NABAVI et al., 2017). O café apresenta uma atividade antioxidante três a cinco vezes superior ao vinho tinto e chás (METRO et al., 2017). Por esta razão, o café é entendido como uma bebida dietética que pode inibir o estresse oxidativo. Os triterpenos e alcaloides como o ácido clorogênico e trigonelina, presentes no café, além das propriedades fisiológicas e farmacológicas que conferem à saúde humana como sequestro de radicais livres e quelação de metais de transição, muitas vezes responsáveis pelo aumento de radicais livres no organismo humano, são conhecidos por contribuir com o sabor e aroma característicos das bebidas de café (FARAH et al., 2007; VIGNOLI et al., 2014; CASTRO et al., 2018). A trigonelina (uma N-metil betaína) também atua no sistema nervoso central, sobre a secreção da bile e a motilidade intestinal (LUDWIG, et al. 2014).

A ingestão excessiva de café, entretanto, pode levar a complicações neurológicas, como diminuição do limiar das atividades convulsivas em indivíduos epiléticos devido a presença da cafeína. A cafeína (1,3,7-trimetilxantina) é um estimulante neurológico encontrado em diferentes tipos de bebidas (chás, cafés, 
refrigerantes) e mais comumente e amplamente ingerido pelo ser humano (REIFMAN, 2014). A história da cafeína está intimamente associada à história do café e seu efeito estimulante no sistema nervoso central são conhecidos desde o século XVII (MITCHELL et al., 2015).

Uma vez que o café, além de ser uma bebida estimulante, possui uma importância social unindo pessoas para uma conversa informal ou profissional, o objetivo deste trabalho foi avaliar se os cafés descafeinados perdem seu potencial antioxidantes ou se apresentam capacidade antioxidantes equivalente aos cafés cafeinados (convencionais ou orgânicos), permitindo que pessoas com doenças neurológicas possam usufruir deste ato social e das propriedades terapêuticas do café, sem provocar danos à saúde.

\section{MATERIAIS DE MÉTODOS}

Esta pesquisa científica tratou-se de um trabalho descritivo experimental seguindo metodologia com testes padronizados internacionalmente. Neste estudo, manipulou-se variáveis independentes para as 13 amostras avaliadas. As amostras de cafés avaliadas foram adquiridas no comércio da cidade de São Paulo. Das 13 amostras estudadas, 10 são cafés cafeinados, sendo sete de cultivo convencional (grupo 01) e três de cultivo orgânico (grupo 02) e três amostras são cafés descafeinados (grupo 03).

As amostras foram preparadas pelo método de infusão tradicional, utilizandose $18,50 \mathrm{~g}$ de pó de café comercial (descafeinado, cafeinado tradicional e orgânico) e $300 \mathrm{~mL}$ de água destilada fervendo. Cada extrato obtido foi resfriado e foram realizadas as diluições.

\section{ENSAIOS}

A atividade antioxidante das soluções de café foi avaliada pela metodologia ABTS de acordo com Souza e colaboradores (2007) e Suriano et al.,(2018). Preparou-se uma solução aquosa $\mathrm{ABTS}^{2-} 2 \mathrm{mM}$ em água deionizada $(18 \mathrm{M} \Omega \mathrm{cm}$ ) e diluiu-se em tampão de fosfato $20 \mathrm{mM}(\mathrm{pH} \mathrm{7,4)}$ até uma concentração final de 100 uM. Alíquotas da solução diluída $(5 \mathrm{~mL})$ foram transferidas para tubos de ensaio contendo $0,0200 \mathrm{~g}$ de $\mathrm{MnO}_{2}$ e agitada durante 50s. As suspensões foram centrifugadas e os sobrenadantes foram filtrados em filtro de seringa de difluoreto de polivinilo (PVDF) 0,22 $\mathrm{m}$ para remover o dióxido de manganês residual. A absorbância (734 nm) do filtrado foi ajustada para 0,90 ( $\pm 0,04)$, o que corresponde a uma concentração final de [ABTS -"] $=60 \mathrm{M}, \mathrm{A}_{\text {ref }}\left(\varepsilon_{734}=1,5 \times 104 \mathrm{M}^{-1} \mathrm{~cm}^{-1}\right)$.

Em cubetas descartáveis de poliestireno foram adicionados $10 \mathrm{uL}$ de soluções de diferentes cafés em diferentes diluições. Em seguida adicionou-se rapidamente 2 $\mathrm{mL}$ da solução $60 \mathrm{M}$ de ABTS ' e monitorou-se as variações de absorbância em função do tempo em $734 \mathrm{~nm}$ por $180 \mathrm{~s}$, conforme exemplificado no Gráfico 1A. As variações de absorbância foram anotadas e determinada a absorbância de referência (A ref.) de cada amostra. Para determinar a porcentagem de radicais ABTS ' sequestrados, $\mathrm{RS}(\%)$, em cada solução, utilizou-se a expressão:

$$
\text { RS }(\%)=[(\text { A ref. }- \text { A amostra }) / \text { A ref. }] \times 100
$$

\section{ANALISE DOS DADOS}

A capacidade antioxidante de remover $50 \%$ dos radicais de $\mathrm{ABTS}^{\circ}, \mathrm{CA}_{50}$, foram obtidos pela equação 1: 
$\mathrm{CA}_{50}=10 \cdot \mathrm{FD}_{50}$.

Equação 1

Para a determinação dos valores de $\mathrm{CA}_{50}$, primeiro foram construídas as curvas de decaimento espectrofotométrico do radical ABTS para cada tipo de café estudado, em diferentes concentrações (fatores de diluição), Gráfico 1A. Uma curva de porcentagem de radicais sequestrados, RS (\%), versus fator de diluição (FD) foi obtida por regressão linear e 0 valor de $\mathrm{FD}_{50}$ determinado pela equação da reta (Gráfico 1B). A partir do valor $\mathrm{FD}_{50}$ para cada café determinou-se a $\mathrm{CA}_{50}$ de cada amostra de café, Equação 1.

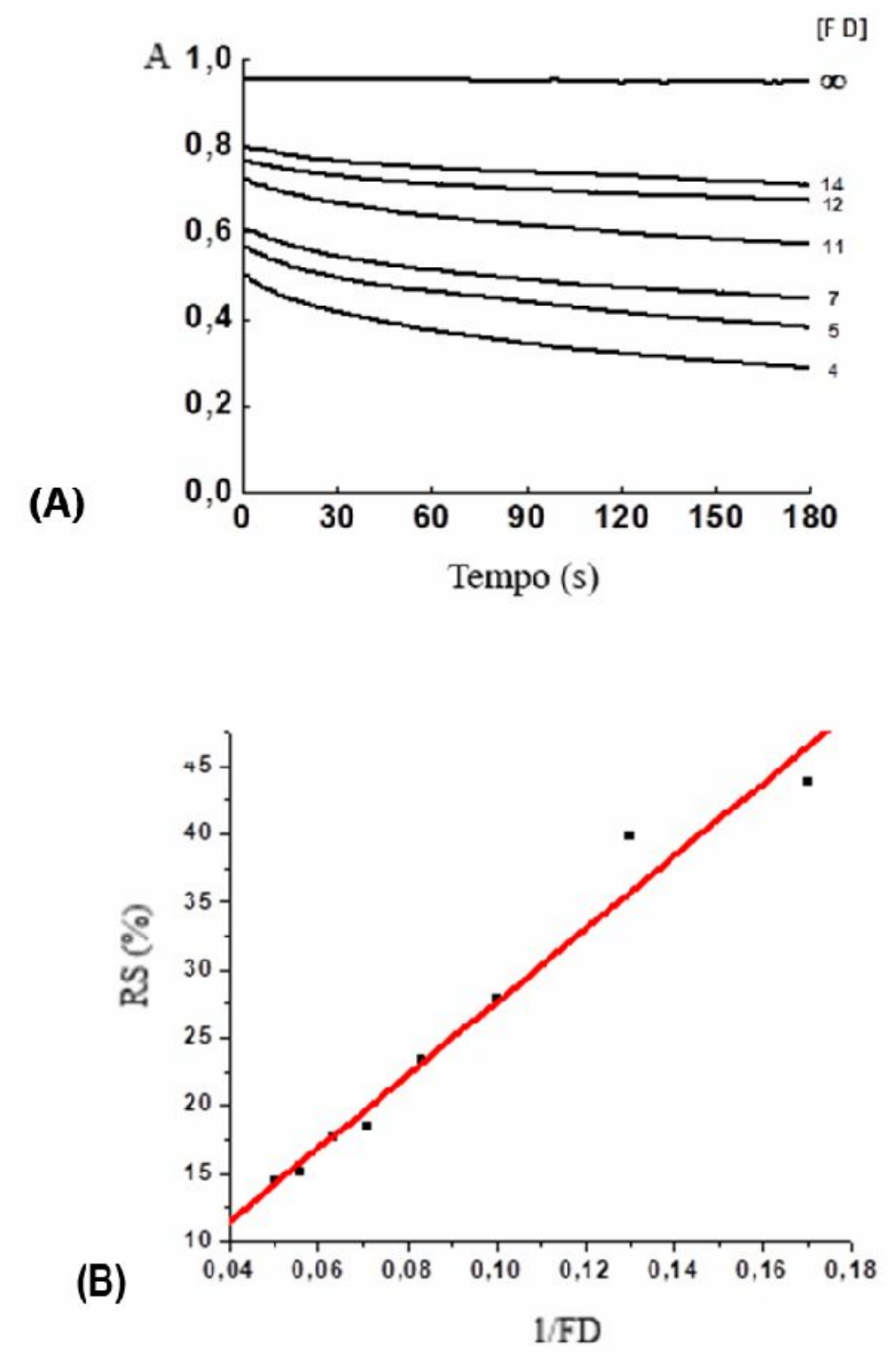

GRÁFICO 1: A) Curvas cinéticas de decaimento do radical ABTS $^{\circ}$ em $735 \mathrm{~nm}$, utilizando-se diferentes diluições (FD) do Café Arauto Cafeinado (C1); (B) Curva de Porcentagem de Radicais de ABTS-- seqüestrados (\% RS) versus Fator de Diluição (FD) para o "Café Arauto Cafeinado (C1). 


\section{ANÁLISE ESTATÍSTICA}

Para comparação da capacidade Antioxidante entre 13 amostras de cafés foi utilizado o teste de análise de variância, utilizando o pós-teste Tukey Kramer e como teste de normalidade o de Kolmogorov e Smirnov. Foi considerado $p<0,05$ como estatisticamente significante.

\section{RESULTADOS}

A capacidade antioxidante (CA) dos cafés analisados foi determinada pelo método ABTS e os resultados obtidos avaliados estatisticamente. Os dados de capacidade antioxidante de supressão de $50 \%$ do radical livre ABTS ${ }^{-}$médio $\left(\mathrm{CA}_{50}\right)$ de todas as amostras de café analisadas com seus respectivos desvios padrão, café convensional (Grupo 01), descafeinado (Grupo 02) e café orgânico (Grupo 03), estão representados na forma de tabela com os repectivos valores de $p$, Tabela 1, e na forma de gráfico com seus respectivos desvios padrão (Gráfico 2).

TABELA 1: Capacidade antioxidante $50 \%$ de supressão o radical ABTS-" das amostras de café e seus respectivos desvios padrão (DP).

\begin{tabular}{|c|c|}
\hline CAFÉS CAFEINADOS - GRUPO 01 & CA 50 \pm DP \\
\hline C1 & $53,0 \pm 2,3$ \\
\hline C2 & $78,8 \pm 4,9^{\mathrm{A}}$ \\
\hline C3 & $66,5 \pm 4,5^{\mathrm{BD}}$ \\
\hline C4 & $79,4 \pm 1,1^{\text {AG }}$ \\
\hline C5 & $62,6 \pm 2,7^{\mathrm{EJ}}$ \\
\hline C6 & $80,6 \pm 2,5^{\text {AGL }}$ \\
\hline C7 & $86,3 \pm 2,7^{\text {AHL }}$ \\
\hline CAFÉS ORGÂNICOS - GRUPO 02 & CA 50 \\
\hline O1 & $76,9 \pm 1,1^{\text {ANQ }}$ \\
\hline O2 & $73,0 \pm 3,0^{\text {APQ }}$ \\
\hline O3 & $76,2 \pm 2,5^{\text {ANQ }}$ \\
\hline CAFÉS DESCAFEINADOS - GRUPO 03 & CA 50 \\
\hline D1 & $38,1 \pm 1,0^{\text {CFIKMOP }}$ \\
\hline D2 & $79,9 \pm 1,4^{\text {AGLQ }}$ \\
\hline D3 & $71,7 \pm 1,8^{\text {APQ }}$ \\
\hline
\end{tabular}

${ }^{A} \mathrm{P}<0,0001$ aumento em relação ao Arauto C1 (ANOVA - One-way - Tukey Kramer); ${ }^{\mathrm{B}} \mathrm{P}<0,001$ aumento em relação ao Arauto C1 (ANOVA - One-way - Tukey Kramer); ${ }^{C} \mathrm{P}<0,001$ redução em relação ao Arauto C1 (ANOVA - One-way - Tukey Kramer); ${ }^{D} \mathrm{P}<0,05$ redução em relação ao Cafeinado C2 (ANOVA - One-way - Tukey Kramer); ${ }^{\mathrm{E}} \mathrm{P}<0,001$ redução em relação ao Cafeinado $\mathrm{C} 2$ (ANOVA - One-way - Tukey Kramer) ${ }^{\mathrm{F}} \mathrm{P}<0,0001$ redução em relação ao Cafeinado C2 ; ANOVA - Oneway - Tukey Kramer); ${ }^{G} \mathrm{P}<0,05$ aumento em relação ao Cafeinado C3 (ANOVA - One-way - Tukey Kramer); ${ }^{\mathrm{H}} \mathrm{P}<0,0001$ aumento em relação ao Cafeinado C3 (ANOVA - One-way - Tukey Kramer); ' $P<0,0001$ redução em relação ao Cafeinado C3 (ANOVA - One-way - Tukey Kramer); ${ }^{\mathrm{P}}<00,001$ redução em relação ao Cafeinado C4 (ANOVA - One-way - Tukey Kramer); ${ }^{\mathrm{K}} \mathrm{P}<0,0001$ redução em relação ao Cafeinado C4 (ANOVA - One-way - Tukey Kramer); ${ }^{\mathrm{L}} \mathrm{P}<0,0001$ aumento em relação ao Cafeinado C5 (ANOVA - One-way - Tukey Kramer); ${ }^{\mathrm{M}} \mathrm{P}<0,0001$ redução em relação ao Cafeinado C5 (ANOVA - One-way - Tukey Kramer); ${ }^{\mathrm{N}} \mathrm{P}<0,0001$ redução em relação ao Cafeinado C5 (ANOVA One-way - Tukey Kramer); ${ }^{\circ} \mathrm{P}<0,0001$ redução em relação ao Cafeinado C6 (ANOVA - One-way Tukey Kramer); ${ }^{P} \mathrm{P}<0,001$ redução em relação ao Cafeinado C7 (ANOVA - One-way - Tukey Kramer); ${ }^{Q_{P}<0,0001}$ aumento em relação ao Arauto Descafeinado D1 (ANOVA - One-way - Tukey Kramer) 
A análise estatistica das amostras de café do grupo 1 (C2-C7) mostrou que as amostras $\mathrm{C} 4, \mathrm{C} 5$ e $\mathrm{C} 7$ apresentaram um aumento $(\mathrm{P} 0,05)$ estatisticamente significativo de $\mathrm{CA}$ quando comparado com a amostra $\mathrm{C}$ 3. Já em relação a $\mathrm{C} 5$, as amostras $\mathrm{C} 6$ e $\mathrm{C} 7$ apresentaram aumento $(P$ 0,0001) extremamente significantes de CA. As amostras de café Arauto, C1 e D1, quando comparados com as demais amostras dos 3 grupos apresentaram uma redução $(P \quad 0,001)$ da CA.

Fazendo-se uma comparação entre os três grupos, sem inclusão dos cafés Arautos (C1 e D1), observa-se que D2 e D3 não apresentaram CA significativamente diferentes dos valores médios de CA das amostras de café tradicionais e orgânicos, com excessão da amostra C7, que apresentou valor superior a todas as amostras dos três grupos (Grafico 2).

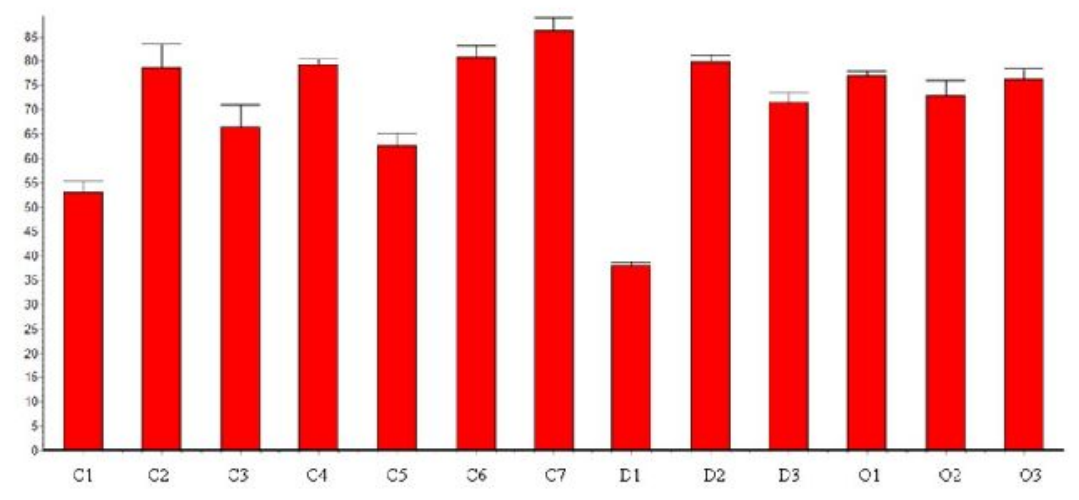

GRÁFICO 2: Capacidade Antioxidante $50 \%$ de supressão do radical ABTS"- médio $\left(\mathrm{CA}_{50}\right)$ das 13 amostras de cafés avaliadas e seu respectivos desvio padrão (DP).

\section{DISCUSSÃO}

Neste estudo foi mostrado que há uma variação estatítica da capacidade antioxidante (CA) entre as diferentes amostras de cafés (Gráfico 2). Das 13 amostras de café avaliadas, foi possível observar que os cafés de cultivo tradicional cafeinados (Grupo 01), cafeinados orgânicos (Grupo 02) e descafeinados (Grupo 03) apresentaram CA estatisticamente diferente quando comparado dentro do mesmo grupo, provavelmente devido aos diferentes processamentos do café no pré e póscultivo, as condições climáticas durante o cultivo, o tipo de solo e o tempo e intensidade de torrefação dos grãos.

Compostos como os ácidos cafeiuquinicos (o ácido clorogênico por exemplo) e os ácidos feruloilquínicos, são compostos fenólicos responsáveis não só pelo sabor e aroma agradáveis do café, bem como pelas suas propriedades antioxidantes (BHATTACHERJEE ; DATTA, 2015). Estes compostos são sensíveis a fatores genéticos, ambientais e condições de manejo pré e pós-colheita, como torrefação, podendo sofrer degradação ou serem produzidos em menor concentração nos grãos de café afetando, assim, a capacidade antioxidante (CA) do café e suas propriedades benéficas à saúde humana.

Já é sabido que a variação na composição química dos grãos de café depende de fatores genéticos, ambientais e condições de manejo pré e pós-colheita (IVAMOTO et al., 2017). Neste trabalho foi mostrado valores muito inferiores de CA dos cafés Arauto, com uma redução significativa $(P \quad 0,001)$ em relação às demais amostras de café, pode estar relacionados com a menor concentração de compostos antioxidantes devido ao cultivo em elevadas altitudes (acima de $1000 \mathrm{~m}$ ). 
Da mesma foram, estudos realizados no sudeste da etiópia mostraram que o cultivo em elevadas altitudes é um fator que também influencia na composição química e nutricional dos grãos de café. A concentração de cafeína e ácido clorogênico, por exemplo, foram afetados pela altitude (WORKU et al., 2018). Os cafés Arauto, portanto, apresentaram CA muito inferior às demais amostras, confirmando que o cultivo em elevadas altitudes pode afetar a concentração de compostos antioxidantes nos grãos de café.

Os cafés descafeinados (com exceção do arauto) não apresentaram diferenças significativas das CAs, quando comparados com a maioria das amostras de cafés contendo cafeina. O processo de descafeinação industrial é realizado nos grãos de café crus inteiros, antes da torrefação. A maioria dos métodos existente utiliza solventes para extração da cafeína, como diclorometano, clorofórmio, álcool, acetona, água e outros. No Brasil, o diclorometano é o mais utilizado (TOCl et al., 2006). Outro processo industrial utilizado em países desenvolvidos, como os Estados Unidos, é a extração por fluido supercrítico de $\mathrm{CO}_{2}$. Este processo utiliza o gás $\mathrm{CO}_{2}$ em alta pressão como solvente, o que permite uma maior eficiência na extração e maior seletividade, extraindo apenas a cafeína, porém é uma técnica de custo elevado (ANDRADE et al., 2012). Diferentes processos de extração podem levar a variações na composição química do café (MULLEN et al., 2011), entretanto, nas amostras analisadas 0 processo de descafeinação não alterou significativamente a CA dos cafés descafeinados, já que os valores de CA encontrados para as amostras de café cafeínado e descafeinado não diferiram estatisticamente.

\section{CONCLUSÕES}

A análise estatística das diferentes amostras (cafés cafeinados e descafeinados) mostrou que a extração da cafeína não é um fator preponderante para a diminuição da capacidade antioxidante (CA) do café. Portanto, pessoas com doenças neurológicas como epiléticos, podem se beneficiar do consumo do café descafeinado, sem perder os benefícios da ingestão da bebida rica em antioxidantes, a qual pode funcionar como um coadjuvante no controle das convulsões.

\section{AGRADECIMENTO}

A universidade anhanguera de são Paulo - UNIAN- SP pelo suporte financeiro.

\section{REFERÊNCIAS}

ANDRADE, K. S.; GONÇALVEZ, R. T.; MARASCHIN, M.; DO VALE, M. R.; MARTÍNEZ, J.; FERREIRA, S. R.S. Supercritical fluid extraction from spent coffee grounds and coffee husks: Antioxidant activity and effect of operational variables on extract composition. Talanta, v. 88, p. 544-552, 2012. https://www.sciencedirect.com/science/article/pii/S0039914011010137> doi: 10.1016/j.talanta.2011.11.031

BHATTACHERJEE, A.; DATTA, A. Mechanism of antiglycating properties of syringic and chlorogenic acids in in vitro glycation system. Food Research International, v. 77 ,

p. 540-548, 
https://www.sciencedirect.com/science/article/pii/S096399691530140X> doi: 10.10 16/j.foodres.2015.08.025

CASTRO, A.C.C.M.; ODA, F.B.; ALMEIDA-CINCOTTO, M.G.J.; DAVANÇO, M.G.; CHIARI-ANDRÉO, B.G. et al. Green coffee seed residue: A sustainable source of antioxidant compounds. Food Chemistry, v. 25, n. 246, p. 48-57, 2018. https://www.ncbi.nlm.nih.gov/pubmed/29291876> doi:10.1016/j.foodchem.2017.10.153

CHUNG YH, KIM WK, KO KH, BACH JH, et al. Role of oxidative stress in epileptic seizures. Neurochemistry International, v. 59, n. 2, p. 122-37, 2011. https://www.ncbi.nlm.nih.gov/pmc/articles/PMC3606551> doi: 10.1016/j.neuint.2011.03.025.

IVAMOTO, S.T.; REIS O JÚNIOR; DOMINGUES, D.S; DOS SANTOS, T. B.; DE OLIVEIRA, F.F.; POT, D.; LEROY, T.; VIEIRA, L.G.; CARAZZOLLE, M.F.; PEREIRA, G.A.; PEREIRA, L.F. Transcriptome Analysis of Leaves, Flowers and Fruits Perisperm of Coffee arabica L. Reveals the Differential Expression of Genes Involved in Raffinose Biosynthesis. PLoS One, v. 12, n. 1: e0169595, 2017. https://journals.plos.org/plosone/article?id=10.1371/journal.pone.0169595. doi: 10.1371/journal.pone.0169595

LUDWIG, I.A.; CLIFFORD M.N.; LEAN, M.E.; ASHIHARA, H.; CROZIER, A. Coffee: biochemistry and potential impact on health. Journal of Functional Foods, v.5, p. 695-717, 2014. https://www.researchgate.net/publication/261138831_Coffee_Biochemistry_and_pot ential_impact_on_health> doi: 10.1039/c4fo00042k

METRO, D.; CERNARO, V.; SANTORO, D.; PAPA, M.; BUENI, M.; BENVENGA, S.; MANASSERI, L. Beneficial effects of oral pure caffeine on oxidative stress. Journal of Clinical and Translational Endocrinology, v. 10, p. 22-27, 2017. https://doi.org/10.1016/j.jcte.2017.10.001>doi: 10.1016/j.jcte.2017.10.001.

MITCHELL, D.C.; HOCKENBERRY, J.; TEPLANSKY, R.; HARTMAN, T.J. Assessing dietary exposure to caffeine from beverages in the U.S. population using brandspecific versus category-specific caffeine values. Food Chem Toxicology, v. 80, p. 247-252,

2015.

https://www.sciencedirect.com/science/article/pii/S0278691515001039> doi:10.016/j.fct.2015.03.024

MULLEN, W.; NEMZER, B.; OU, B.; STALMACH, A.; HUNTER, J.; CLIFFORD, M.N.; COMBET E. The Antioxidant and Chlorogenic Acid Profiles of Whole Coffee Fruits Are Influenced by the Extraction Procedures. Journal of Agricultural and Food $\begin{array}{lllll}\text { Chemistry, } & \text { v. } & 59, & \text { p. } & \text { 3754-3762, }\end{array}$ https://pubs.acs.org/doi/abs/10.1021/jf200122m?journalCode=jafcau.> doi: $10.1021 / \mathrm{jf200122m}$

NABAVI, S.F.; TEJADA, S.; SETZER, W.N.; GORTZI, O.; SUREDA, A.; BRAIDY, N.; DAGLIA, M.; MANAYI A.; NABAVI, S.M. Chlorogenic Acid and Mental Diseases: 
From Chemistry to Medicine. Current Neuropharmacology, v. 15, n. 4, p 471-479, 2017. https://www.ncbi.nlm.nih.gov/pmc/articles/PMC5543670>doi: 10.2174/1570159X14666160325120625.

NIEMAN, D. C.; GOODMAN, C. L.; CAPPS, C. R.; SHUE, Z. L.; ARNOT, R. Influence of 2-Weeks Ingestion of High Chlorogenic Acid Coffee on Mood State, Performance, and Postexercise Inflammation and Oxidative Stress: A Randomized, Placebo-Controlled Trial. International Journal of Sport Nutrition and Exercise Metabolism. $\quad$ v. 28, n. 1, p. 2018. https://www.researchgate.net/journal/1526484X_International_journal_of_sport_nutrit ion_and_exercise_metabolism. doi:10.1123/ijsnem.2017-0198

PRASAD, D.K.; SATYANARAYANA, U.; HAHEENS, U.; SURYA PRABHA, T.; MUNSHI, A. Oxidative Stress in the Development of Genetic Generalised Epilepsy: An Observational Study in Southern Indian Population. Journal of Clinical and Diagnostic Research. v. 11, n. 9, BC05-BC08, 2017. https://www.ncbi.nlm.nih.gov/pmc/articles/PMC5713714>doi:10.7860/JCDR/2017/29 133.10604.

PUTTACHARY, S.; SHARMA, S.; STARK, S.; THIPPESWAMY, T. Seizure-induced oxidative stress intemporal lobe epilepsy. BioMed Research International, v. 2015, ID 745613, 2015. http://dx.doi.org/10.1155/2015/745613 > doi: $10.1155 / 2015 / 745613$.

REIFMAN, J. Dose-dependent model of caffeine effects on human vigilance during total sleep deprivation. Journal of Theoretical Biology, v. 7, n. 358, p. 11-24, 2014. https://www.ncbi.nlm.nih.gov/pubmed/24859426> doi: 10.1016/j.jtbi.2014.05.017.

SHICHIN, M. The role of lipid peroxidation in neurological disorders. ournal of Clinical Biochemistry and Nutrition, v.54, n.3, p.151-60, 2014. https://www.ncbi.nlm.nih.gov/pmc/articles/PMC4042144>doi: 10.3164/jcbn.14-10.

SOUZA, C.M.M., SILVA, H.R.; VIEIRA-JUNIOR G.M.; AYRES, C.L.S.C; ARAUJO,

D.S. Fenóis totais e atividade antioxidante de cinco plantas medicinais. Química Nova, v30, n.2, p.351-355, 2007. http://dx.doi.org/10.1590/S010040422007000200021> doi:10.1590/S0100-40422007000200021.

SURIANO, S.; IANNUCCI, A.; CODIANNI, P.; FARES, C.; RUSSO, M.; PECCHIONI, N.; MARCIELLO, U.; SAVINO, M. Phenolic acids profile, nutritional and phytochemical compounds, antioxidant properties in colored barley grown in southern Italy. Food Research International. v. 113, p. 221-233, 2018. https://www.sciencedirect.com/science/article/pii/S0963996918305180>doi:10.1016/j. foodres.2018.06.072

TOCI, A.; FARAH, A.; TRUGO, L.C. Efeito do processo de descafeinação com diclorometano sobre a composição química dos cafés arábica e robusta, antes e 
após a torração. Química nova, v.29, p.965-971, 2006. http://www.quimicanova.sbq.org.br/imagebank/pdf/Vol29No5_965_14-AR05334.pdf>

VIGNOLI, J.A.; VIEGAS, M.C.; BASSOLI, D.G.; BENASSI, M.T. Roasting process affects differently the bioactive compounds and the antioxidant activity of Arabica and Robusta coffees. Food Research International, v.61, p.279-285, 2014. https://www.sciencedirect.com/science/article/pii/S0963996913003335>doi:10.1016/j. foodres.2013.06.006.

WORKU, M; DE MEULENAER, B; DUCHATEAU, L; BOECKX, P. Effect of altitude on biochemical composition and quality of green arabica coffee beans can be affected by shade and postharvest processing method. Food Research International, v. 105, $\quad$ p. 278-285, 2018. https://doi.org/10.1016/j.foodres.2017.11.016>doi: 10.1016/j.foodres.2017.11.016.

YESIL, A.; YILMAZ, Y. Review article: Coffee consumption, the metabolic syndrome and non-alcoholic fatty liver disease. Aliment. Pharmacology and Therapeutics, v.38, n.9, p.1038-1044, 2013. https://onlinelibrary.wiley.com/doi/epdf/10.1111/apt.12489> doi: 10.1111/apt.12489. 